Meta

Journal des traducteurs

Translators' Journal

\title{
Proverbes : transparence et opacité
}

\section{Georges Kleiber}

Volume 55, numéro 1, mars 2010

Le parcours du sens : d'une langue à l'autre — Mélanges offerts à André Clas

The Way of Meaning: From a Language to Another - Collection of Articles Offered to André Clas

URI : https://id.erudit.org/iderudit/039608ar

DOI : https://doi.org/10.7202/039608ar

Aller au sommaire du numéro

\section{Éditeur(s)}

Les Presses de l'Université de Montréal

ISSN

0026-0452 (imprimé)

1492-1421 (numérique)

Découvrir la revue

Citer cet article

Kleiber, G. (2010). Proverbes : transparence et opacité. Meta, 55(1), 136-146. https://doi.org/10.7202/039608ar

\section{Résumé de l'article}

Notre contribution a pour objectif d'examiner la question de la transparence des proverbes, tout particulièrement celle des proverbes métaphoriques. Dans une première partie, nous exposerons en quoi consiste exactement la transparence sémantique assignée aux proverbes métaphoriques; dans la seconde, après avoir rappelé notre approche hiérarchique des proverbes, nous montrerons comment le statut superordonné des proverbes a comme corollaire le maintien de la transparence du sens littéral ou compositionnel. Chemin faisant, se confirmera la justesse d'un traitement des proverbes comme une catégorie sémantique en soi.
Ce document est protégé par la loi sur le droit d'auteur. L'utilisation des services d’Érudit (y compris la reproduction) est assujettie à sa politique d'utilisation que vous pouvez consulter en ligne.

https://apropos.erudit.org/fr/usagers/politique-dutilisation/ 


\title{
LEXIQUE
}

\section{Proverbes: transparence et opacité}

\author{
GEORGES KLEIBER \\ Université de Strasbourg, Strasbourg, France \\ kleiber@umb.u-strasbg.fr
}

\begin{abstract}
RÉSUMÉ
Notre contribution a pour objectif d'examiner la question de la transparence des proverbes, tout particulièrement celle des proverbes métaphoriques. Dans une première partie, nous exposerons en quoi consiste exactement la transparence sémantique assignée aux proverbes métaphoriques; dans la seconde, après avoir rappelé notre approche hiérarchique des proverbes, nous montrerons comment le statut superordonné des proverbes a comme corollaire le maintien de la transparence du sens littéral ou compositionnel. Chemin faisant, se confirmera la justesse d'un traitement des proverbes comme une catégorie sémantique en soi.
\end{abstract}

\begin{abstract}
Our contribution examines the question of the transparency of proverbs, particularly metaphorical proverbs. In a first part, we will present what exactly the semantic transparency of metaphorical proverbs consists in; in the second, after recalling our hierarchical approach to proverbs, we will demonstrate how the superordinate status of proverbs helps to preserve the transparency of the literal or compositional meaning. This sets the stage to confirm the treatment of proverbs as a semantic category in itself as the right approach.
\end{abstract}

\section{MOTS-CLÉS/KEYWORDS}

proverbes, transparence/opacité, figement, sémantique des proverbes, métaphores proverbs, transparency/opacity, fixation, semantics of proverbs, metaphors

À André Clas

\section{Introduction}

La sémantique des proverbes a le vent en poupe et le pessimisme définitoire d'un Taylor ${ }^{1}$ semble bien loin. Les nombreuses études ${ }^{2}$ parues durant ces vingt dernières années établissent en effet, plus ou moins nettement, que les proverbes représentent un objet sémantique spécifique et qu'il est donc légitime d'essayer de les définir sémantiquement. Le problème, c'est que les analyses proposées sont loin de concorder. Certaines peuvent être aux antipodes les unes des autres ${ }^{3}$, d'autres divergent fortement sur telle ou telle caractéristique prêtée aux proverbes. S’agit-il ou ne s'agit-il pas de dénominations? Faut-il ou non parler de phrases génériques à leur propos ${ }^{4}$ ? Fontelles partie ou non des expressions figées? Le débat sur la nature sémantique des proverbes n'est pas clos, loin de là.

Nous le poursuivrons ici en examinant la question de la transparence ou de l'opacité sémantique des proverbes. Anscombre (2003) et, tout récemment, Tamba (à 
paraître $)^{5}$ ont montré que les proverbes dits métaphoriques n'étaient pas opaques comme le sont les expressions idiomatiques. Pour Anscombre, la raison en est le caractère non figé des proverbes. Pour Tamba, c'est la particularité, par rapport au sens idiomatique, du sens proverbial, compris comme le résultat du couplage de deux sens complets autonomes, l'un compositionnel et l'autre formulaire, qui est à l'origine de cette transparence sémantique. Nous apporterons ici un prolongement explicatif à leurs conclusions en essayant de montrer que les deux principales données avancées pour appuyer la transparence des proverbes s'expliquent, l'une, par leur statut de phrase, et l'autre, par leur hauteur abstractive, que nous avons postulée comme étant l'un de leurs traits définitoires (Kleiber 2008; à paraître).

Notre analyse se fera en deux parties. La première exposera en quoi consiste exactement la transparence sémantique assignée aux proverbes métaphoriques; la seconde, après avoir rappelé notre approche hiérarchique des proverbes, montrera comment le statut superordonné des proverbes a comme corollaire le maintien de la transparence du sens littéral ou compositionnel. Chemin faisant, se confirmera la justesse d'un traitement des proverbes comme une catégorie sémantique en soi.

\section{Proverbes métaphoriques et transparence sémantique}

Pour éviter toute équivoque, quelques précisions sont nécessaires au préalable sur ce que l'on entend par transparence ou opacité des proverbes. Il y a en effet plusieurs paliers d'application possibles de ces notions aux proverbes: sur le plan dénominatif, sur le plan du sens descriptif et, en ce qui concerne les proverbes métaphoriques, sur le plan de la subsistance du sens littéral dans sa relation avec le sens proverbial.

\subsection{Premier niveau: dénominatif}

Il nous faut commencer par la question de la dénomination. Si l'on accepte que les proverbes sont des dénominations, alors tous les proverbes seront déclarés sémantiquement opaques, dans la mesure où toute combinaison polylexicale qui représente une dénomination (noms composés, expressions figées ${ }^{6}$ ) donne lieu à un sens global qui répond à la définition de l'opacité sémantique ${ }^{7}$, puisque ce sens global n'est pas le résultat de la combinaison du sens des constituants lexicaux et de la structure syntaxique.

Rappelons brièvement pourquoi. Une dénomination (quelle qu'elle soit) comporte comme nous l'avons défendu ailleurs (Kleiber 2001 ; 2002; 2003a; 2003b) deux composants sémantiques:

(1) une partie commune à toutes les dénominations et qui indique qu'il s'agit d'une chose, ou si l'on veut, d'un tout;

(2) une partie qui varie avec les dénominations et qui consiste en la description ou représentation du type de choses dont il s'agit. Il s'ensuit une situation paradoxale, que nous avons appelée le paradoxe sémantique de la dénomination, qui réside dans l'impossibilité a priori pour la partie descriptive d'exprimer totalement le sens d'une dénomination. La partie (1) du sens se trouve marquée iconiquement par l'unité formelle qui caractérise une dénomination ${ }^{8}$.

Notre analyse prédit que le sens d'une unité polylexicale ${ }^{9}$ n'est jamais (totalement) un sens compositionnel, puisque la seule combinaison des sens des constituants ne 
peut donner accès à la partie (1), c'est-à-dire au composant sémantique directement lié au statut de dénomination, qui indique qu'il s'agit d'un type ou d'une catégorie de choses. Prenons fauteuil roulant ${ }^{10}$ en tant que nom composé, c'est-à-dire en tant que dénomination: si son sens était seulement compositionnel, on devrait obtenir un fauteuil roulant en mettant des roulettes sous les pieds d'un fauteuil, mais on n'obtient qu'un fauteuil... avec des roulettes. Fauteuil roulant, en tant que dénomination, renvoie à une classe restreinte de fauteuils, ceux qui servent aux handicapés à se déplacer.

On comprend ainsi pourquoi leur statut dénominatif rend tous les proverbes opaques, les littéraux, tels que:

- Qui peut le plus peut le moins;

- L'union fait la force;

comme les métaphoriques, tels que:

- Pierre qui roule n'amasse pas mousse;

- Chat échaudé craint l'eau froide.

La combinaison sémantique des seuls constituants ne peut en effet donner lieu au correspondant sémantique qu'amène leur statut de dénomination. On peut toutefois s'interroger sur la nature de cette contrepartie sémantique, surtout à propos des proverbes littéraux, qui présentent un sens qui semble peu ou prou être celui de la combinaison du sens de leurs constituants. Quel sens supplémentaire, non compositionnel, est apporté par le facteur dénominatif du proverbe L'union fait la force? La comparaison avec les phrases génériques livre la réponse. Si L’union fait la force est considérée comme une simple phrase générique et non comme un proverbe, il s'agit d'une vérité qui se trouve uniquement assertée. En tant que proverbe, cette vérité est déjà acquise par le statut dénominatif. Nous avions ainsi écrit (Kleiber 1989: 248): «Avec le proverbe, la dénomination a pour conséquence de présupposer la vérité de la situation générique dénotée et donc de nous dispenser de l'asserter : c'est en cela qu'elle est une vérité universelle, une vérité "pour tout locuteur"11».

\subsection{Deuxième niveau: représentationnel ou descriptif}

Si l'on s'en tenait au seul niveau dénominatif, il n'y aurait pas de différence de transparence entre les proverbes littéraux et les proverbes métaphoriques, puisque, vus sous cet angle, tous sont opaques. Or, il saute aux yeux qu'on ne peut en rester là et mettre sur le même plan des proverbes comme L'union fait la force et Chat échaudé craint l'eau froide. Lorsqu'on applique la notion de transparence à la composante sémantique descriptive, celle qui est propre à chaque proverbe et qui les distingue l'un de l'autre, les proverbes dits littéraux apparaissent plutôt comme transparents et les métaphoriques plutôt comme opaques. Le sens proverbial descriptif de L'union fait la force peut être considéré comme résultant de la composition des sens de ses constituants, c'est la raison pour laquelle on peut aussi parler de proverbes componentiels, alors qu'il n'en va plus ainsi de celui de Chat échaudé craint l'eau froide. Tous les commentateurs ont noté ce phénomène. Les littéraux donnent lieu à un sens représentationnel ou descriptif qui résulte de la composition des sens de leurs constituants, alors que les métaphoriques présentent, tout comme les expressions idiomatiques telle casser sa pipe, un sens qui n'est plus le résultat de la combinaison 
sémantique de leurs parties. Si l'on procède par composition, on obtient un sens compositionnel ou littéral, fort différent de leur véritable sens, que l'on appelle parfois sens figé pour le séparer du sens acquis par composition.

Expressions idiomatiques ${ }^{12}$ et proverbes métaphoriques vont donc de pair à ce niveau représentationnel, en rendant accessibles deux sens, un sens compositionnel ou littéral, qui n'est pas le leur, et un sens qui est le leur, qui s'avère non compositionnel et qui donc est dit opaque ou figé, sens lexicalisé ou conventionnel, qui doit être appris comme étant le sens de la dénomination idiomatique ou proverbiale. Mais sont-ils pour autant opaques de la même manière?

\subsection{Proverbes métaphoriques: subsistance du sens compositionnel}

Anscombre (2003) et Tamba (2000; à paraître) pensent qu'ils ne sont pas opaques. Leurs analyses sont fort différentes - Anscombre argumente pour le caractère non figé des proverbes en général, alors que Tamba leur conserve le statut d'expression figée et plaide pour un sens proverbial qui serait la conjonction du sens compositionnel et du sens formulaire ${ }^{13}$ - mais elles se rejoignent sur deux phénomènes intéressants, qui font jouer l'opposition transparence/opacité sur le plan du rapport entre le sens littéral et le sens de l'expression idiomatique ou celui du proverbe. Ces deux phénomènes les conduisent tous deux à postuler la transparence des proverbes par rapport aux expressions idiomatiques. L'idée sous-jacente, explicite chez Tamba, implicite chez Anscombre, est que la relation entre le sens compositionnel et le sens formulaire d'un proverbe métaphorique ne fait pas disparaître pour autant le sens compositionnel: celui-ci reste ainsi transparent, alors que la relation entre le sens littéral et le sens idiomatique d'une expression idiomatique entraîne la mise sous boisseau du sens compositionnel au profit du seul sens idiomatique (casser sa pipe). Dit d'une autre manière encore, le sens compositionnel ou littéral d'un proverbe métaphorique subsiste avec le sens proverbial, alors que dans le cas d'une expression idiomatique, il se trouve oblitéré par le sens idiomatique: «Le sens idiomatique [...] ne peut coexister avec le sens compositionnel d'une expression» (Tamba, à paraître).

Le premier phénomène qui prouve cette subsistance transparente du sens compositionnel d'un proverbe et l'opacité oblitérante de ce même sens chez les expressions idiomatiques est la nécessité d'adapter la forme du sens littéral d'un proverbe pour assurer la compréhension et non celle du sens littéral d'une expression idiomatique. Anscombre (2003: 167) note ainsi, pour les proverbes, que «si l'interprétation d'une unité lexicale ou d'une construction n'est plus possible à une étape donnée du système linguistique, l'unité lexicale est remplacée par une autre, et la construction syntaxique modifiée». À quoi fait écho cette assertion de Tamba selon laquelle la conjonction des deux sens d'un proverbe métaphorique «pousse à modifier la formule proverbiale au fur et à mesure que l'évolution d'une langue risquerait de rendre inintelligible le sens compositionnel compromettant ainsi sa relation à la loi, principe ou norme, véhiculée par le proverbe» (Tamba, à paraître).

Anscombre (2003: 167) illustre le changement d'unité lexicale en notant que la disparition du verbe s'empeechier avec le sens de 'se freiner', 's'arrêter' a amené le proverbe ancien :

- Qui trop se haiste si s'empeeche 
à devenir :

- Qui trop se hâte reste en chemin.

Il recourt au couple:

- Tout vient à point qui sait attendre;

- Tout vient à point à qui sait attendre;

pour montrer que la perte d'une construction syntaxique, en l'occurrence le tour où qui pouvait s'interpréter comme si... on, entraîne également un changement de construction. Rien de tel avec les expressions idiomatiques. Comme le souligne Anscombre (2003: 167-168), «dans avoir maille à partir, chercher noise, rester coi, boute-en-train, les mots maille, noise, coi, boute ont disparu du français contemporain au sens qu'ils ont dans ces locutions, mais les locutions n'ont pas été modifiées pour autant».

On voit quel est le raisonnement sous-jacent à cette observation: même si le sens compositionnel ne donne pas directement le sens du proverbe métaphorique - ce en quoi on peut, comme nous l'avons vu, le taxer d'opaque -, la nécessité de modifier la forme si l'interprétation littérale risque d'échouer, par suite d'opacité due aux changements diachroniques, est le signe que cette forme doit rester d'une certaine manière interprétable compositionnellement, c'est-à-dire doit, dans son rapport avec le sens proverbial, garder une certaine transparence littérale. On ne peut comprendre le sens proverbial sans comprendre le sens littéral, alors que semblable relation n'est pas nécessairement de mise avec les expressions idiomatiques ${ }^{14}$.

Avec la deuxième donnée, on passe du changement diachronique intralinguistique au changement interlinguistique. Tamba (à paraître) observe qu'on ne peut «traduire littéralement un idiome ${ }^{12}$ d'une langue dans l'autre, alors qu'on peut traduire le sens compositionnel littéral d'un proverbe, à condition d'expliciter quel sens formulaire doit lui être analysé». Anscombre (2003: 168) formule à peu près la même idée en mettant l'accent sur la possibilité, au sein d'un type de culture donné, de comprendre un proverbe d'une autre langue, alors que semblable intercompréhension ne semble pas l'apanage des expressions idiomatiques. Il note ainsi que

L'avenir appartient à celui qui se lève tôt a pour correspondant allemand Morgen hat Gold im Mund. The early bird catches the worm (anglais), Qui matina fa farina (catalan), A quien madruga, Dios le ayuda (espagnol) qui sont immédiatement comprises par les sujets parlants (Anscombre 2003: 168).

Il souligne

[qu'i]l y a même des cas où un proverbe est quasiment identique dans toutes les langues d'un groupe: Une hirondelle ne fait pas le printemps / Una golondrina non hace verano (espagnol) / Una orenata no fa estiu (catalan) / Una rondine non fa primavera (italien) / One swallow does not make a summer (anglais), Eine Schwalbe macht keinen Sommer (allemand) (Anscombre 2003: 168).

Semblables correspondances sont rares avec les expressions figées. Anscombre (2003: 169) se demande ainsi :

Qui pourrait deviner que le français se payer la tête, l'allemand sich über jemand lustig machen, l'anglais to pull one's leg et l'espagnol tomar el pelo sont des tournures grosso modo de même sens? Ou encore casser sa pipe, ins Gras beissen, to kick the bucket, estirar la pata, fer l'ànec? 
Cette seconde donnée va dans le même sens que la première: la compréhension et la traduction d'un proverbe métaphorique d'une autre langue mettent bien évidemment en jeu le sens proverbial, mais continuent de le relier au sens compositionnel. Celui-ci ne disparaît pas dans l'opération: il reste de la sorte transparent.

\section{Quelques pas de plus}

La mise au jour de ces deux phénomènes, la subsistance du sens compositionnel et sa traductibilité, constitue une avancée consistante dans la description sémantique des proverbes métaphoriques. Deux questions se posent tout naturellement: Sont-ils vraiment propres aux proverbes? Comment les expliquer? Nous avons déjà vu que la réponse à la première question est positive pour Anscombre et Tamba. Pour ce qui est de celle à la seconde, nous avons également déjà mentionné qu'Anscombre expliquait la transparence des proverbes tout simplement par le fait qu'il ne s'agit pas d'expressions figées, alors que Tamba y voit la conséquence du couplage du sens formulaire et du sens compositionnel, couplage qui débouche sur le sens proverbial.

\subsection{Modification, proverbes et phrases figées}

Nous reprendrons tout d'abord la première question. Il nous semble que le premier des deux phénomènes, celui de la modification diachronique, ne concerne pas spécifiquement les proverbes, mais s'applique aussi aux expressions figées phrastiques telles que:

- Les carottes sont cuites;

- Le jeu n'en vaut pas la chandelle;

- Un ange passe;

- La messe est dite.

Il n'y a, à notre connaissance, pas de phrases figées (non proverbiales) qui présenteraient une structure lexico-syntaxique ininterprétable, donc opaque de ce point de vue. C'est dire aussi qu'il faut trouver alors une autre explication que celle qui s'appuie sur le proverbe. Nous suggérerons que le facteur qui est à l'origine de la nécessité d'adapter aussi bien proverbes qu'expressions phrastiques figées est d'ordre catégoriel. C'est le statut de phrase commun aux expressions figées phrastiques et aux proverbes qui nous semble décisif. La raison des modifications adaptatives relevées est qu'il faut pouvoir reconnaître aussi bien du point de vue de la construction syntaxique que de celui de la combinaison sémantique de quelle phrase il s'agit. Il ne faut donc pas qu'il y ait, sous cet angle, opacification. Ou, dit autrement, ce qui doit toujours transparaître ou être transparent, c'est le statut de phrase.

On peut se demander pourquoi il n'en va pas ainsi avec les simples expressions figées. Pourquoi peuvent-elles comporter des constituants locaux qui ne sont plus transparents ou interprétables littéralement, car relevant d'un état de langue passé, alors que cela n'est plus de mise avec les phrases figées (proverbiales ou non)? La raison en est, nous semble-t-il, la différence, à l'égard de l'opposition transparence/ opacité, entre les correspondants catégoriels non figés des phrases figées (non proverbiales et proverbiales) et des expressions figées (non phrastiques). Le correspondant des premières est une phrase; à ce titre, il est toujours transparent; celui des secondes représente une unité lexicale, et non plus une expression polylexicale; à ce titre, il 
est, lui, opaque. Prenons l'expression figée casser sa pipe. Elle a pour correspondant non figé l'unité lexicale mourir, qui, faut-il le rappeler, est, bien entendu, arbitraire ou opaque. Si l'on prend par contre Les carottes sont cuites: quel que soit le correspondant non figé qu'on lui attribue (C'est foutu / La situation est désespérée), il s'agit d'une phrase qui n'est pas opaque, et dont l'interprétation se calcule à partir de la combinaison du sens de ses constituants.

\subsection{Traductibilité et intercompréhension}

Le deuxième phénomène, celui de la subsistance du sens compositionnel dans les proverbes métaphoriques, semble, lui, bien spécifique des proverbes, mais, comme il ne concerne véritablement que les proverbes métaphoriques (les autres ne présentant pas deux sens), l'explication d'Anscombre n'arrive pas vraiment à en rendre compte.

Celle de Tamba nous semble beaucoup plus appropriée, car elle met sur la table les bons ingrédients, à savoir le sens compositionnel ou littéral et le sens conventionnel fixé par le proverbe, qu'elle appelle sens formulaire. Nous la suivons un peu moins dans sa décision de ne pas considérer ce sens formulaire comme étant le sens même du proverbe. Elle opte en effet pour une combinaison du sens compositionnel et du sens formulaire qui a pour résultat, selon elle, l'émergence du véritable sens proverbial: «Le sens proverbial résulte de la conjonction stable de deux sens qui, pris séparément, sont autonomes et complets» (Tamba, à paraître). C'est dire que le sens formulaire n'est pas le véritable sens du proverbe, ce n'est qu'une composante de ce sens qui, avec le sens littéral, forme un troisième sens, le sens proverbial. Cela permet d'expliquer, comme on l'a vu ci-dessus, que le sens littéral reste transparent dans le sens proverbial, puisqu'il en est une partie nécessaire. Il y a toutefois une difficulté, c'est que cette solution oblige à postuler trois sens pour les proverbes métaphoriques, le sens compositionnel, le sens formulaire et le sens proverbial. Or, il est difficile de voir en quoi consiste exactement ce troisième sens, ceci d'autant plus que ce n'est pas lui qui se trouve lexicalisé ou conventionnalisé, mais le sens formulaire ${ }^{15}$.

Si l'on peut se passer d'un tel troisième homme, cela nous semble nettement préférable. Une telle solution est possible à condition de voir dans les proverbes, comme nous l'avons fait ailleurs (Kleiber 2008; à paraître a) des dénominations qui catégorisent des catégories, non de niveau basique ou subordonné (Kleiber 1990; 1994b), mais de niveau superordonné, autrement dit, des catégories qui regroupent des situations hétérogènes. Que l'on considère les proverbes à sens non littéral comme les proverbes à sens dit littéral, on constate en effet qu'il est impossible de les représenter par une situation qui refléterait toute la catégorie dénommée par le proverbe. Dès que l'on essaie d'illustrer le sens de proverbes littéraux comme:

- L’union fait la force;

- À petites causes, grands effets;

c'est un exemple de situation particulière qui vient à l'esprit et non une situation qui résumerait la catégorie de situations dénommée par le proverbe. Il en va de même avec les proverbes du type non littéral comme:

- Chat échaudé craint l'eau froide;

- C'est en forgeant que l'on devient forgeron. 
L'illustration de la catégorie de situations dénommée aboutit à se représenter une sous-catégorie ou une instance particulière de cette catégorie seulement. Les proverbes constituent ainsi une catégorisation de situations fort différentes, qui a priori n’apparaissent pas comme étant unies, homogènes. C'est le proverbe, grâce à la dénomination catégorielle de situations qu'il représente, qui opère leur réunion, par le biais d'un lien abstractif général ${ }^{16}$ commun aux situations ainsi rassemblées. Les situations qui forment la catégorie qu'il dénomme, sont, elles, des situations que l'on peut appeler basiques si l'on entend souligner par là que tout comme les noms basiques tels que pomme, chaise, etc., elles se laissent représenter de façon homogène, dans la mesure où elles mettent aux prises des entités et des actions pouvant être représentées par une image abstraite ou concrète commune, reflétant la catégorie. Si nous prenons le proverbe C'est en forgeant que l'on devient forgeron, il s'applique à des situations mettant en jeu des activités particulières basiques dont chacune a son propre schéma ou forme (apprentissage du tennis, de la frappe à l'ordinateur, etc.), mais qui ne se laissent guère résumer par une forme ou un schéma unique qui correspondrait à la catégorie activité ou spécialité.

Si l'on accepte notre hypothèse, on peut expliquer directement pourquoi les proverbes métaphoriques, à la différence des expressions idiomatiques phrastiques, sont traductibles ou compréhensibles littéralement, ou compositionnellement, d'une langue à l'autre. Leur sens littéral, parce que phrastique, est transparent comme celui des phrases figées (voir plus haut), mais il s'accompagne d'une condition d'hyponymie par rapport au sens proverbial (superordonné ou hyperonymique), qui fournit une clé interprétative à toute phrase reconnue comme proverbe métaphorique. C'est ce point fondamental de la sémantique des proverbes qui explique que l'on puisse traduire directement les proverbes métaphoriques, mais non les expressions idiomatiques: l'ascension vers le sens hyperonymique ou superordonné subsiste lorsqu'on traduit le sens littéral d'un proverbe métaphorique, alors que semblable moteur interprétatif n'existe pas dans le cas des phrases figées: il n'y a pas de clé pour accéder à l'interprétation voulue de Un ange passe si on le traduit en allemand, alors que si on traduit Chat échaudé craint l'eau froide dans une autre langue, l'ascenseur hypo/ hyperonymique peut fonctionner pour accéder au sens du proverbe. C'est ce point aussi, avec d'autres (voir le rapport implicatif), qui explique que l'on puisse comprendre un proverbe métaphorique que l'on n'a jamais entendu auparavant. Tout ceci suppose, bien entendu, que l'on accepte que les proverbes forment une catégorie linguistique spéciale, aussi bien sémantique que - étant donné le côté dénominatif - formelle ${ }^{17}$. Ce qui est notre credo depuis de nombreuses années, credo qui, on l'aura sans doute remarqué, demande encore beaucoup d'éclaircissements, d'ajustements, de rectifications et donc... d'investigations!

\section{Conclusion}

Nous pouvons néanmoins conclure. Il nous semble en effet avoir répondu aux principales questions que soulève l'application de l'opposition transparence / opacité aux proverbes et, plus particulièrement, nous avons répondu aux problèmes posés par les deux phénomènes mis en relief par Anscombre (2003) et Tamba (à paraître). La nécessité d'adaptation synchronique s'explique par le statut de phrase des proverbes; la traductibilité littérale des proverbes métaphoriques, qui prouve la subsistance ou 
transparence de leur sens compositionnel, a pour origine la contrainte d'abstractivité catégorielle qui exige que les proverbes se déploient à un niveau d'hétérogénéité semblable à celui des catégories superordonnées.

\section{NOTES}

1. "The definition of a proverb is too difficult to repay the undertaking» (Taylor 1931:3).

2. Voir entre autres les travaux d'Anscombre, Carel et Schultz, Conenna, Gouvard, Honeck, Kleiber, Lakoff et Turner, Mejri, Michaux, Palma, Perrin, Schapira, Tamba, Visetti et Cadiot cités en bibliographie.

3. Un exemple suffira: l'approche de Visetti et Cadiot (2006) est totalement à l'opposé de celle que l'on trouve chez Anscombre ou que nous promouvons nous-même.

4. Dénomination et généricité (au sens de phrase générique) restent sujets à discussion. Tamba (2000: 118) rejette «les solutions dénominative et générique [parce qu’elles] ont le défaut d'éluder, sans la résoudre, la duplicité sémantique foncière du proverbe».

5. Voir aussi Gross (1996: 7): «Les proverbes, les chansons, les poèmes sont aussi des suites qui nous sont imposées: nous ne pouvons pas en changer les éléments, mais nous ne dirons pas que ce sont des suites linguistiquement figées, car leur sens est transparent».

6. Voir Gross (1996) et Mejri (1997).

7. Voir Gross (1996: 10): dans le cas où le sens d'une suite «n'est pas fonction de celui des éléments constitutifs, [...] nous dirons que nous sommes en présence d'une suite opaque ou sémantiquement figée».

8. La rigidité interne ou le degré de figement des unités polylexicales provient de la nécessité de marquer formellement la partie sémantique informant qu'il s'agit d'une catégorie de choses. La relative fixité interne présentée a en effet pour conséquence de conférer à l'ensemble les limites réclamées par un tout.

9. Il en va de même du sens des mots construits.

10. On n'a pas dans ce cas *un fauteuil très roulant.

11. Nous maintenons que les proverbes n'expriment pas des vérités a priori, mais que c'est le statut dénominatif du proverbe qui transforme une vérité «pour un locuteur» en vérité "pour tout locuteur» (voir Perrin pour une position identique et Anscombre, pour une position opposée).

12. Tamba (à paraître) parle dans ce cas d'idiomes.

13. «Autrement dit, le sens proverbial résulte de la conjonction stable de deux sens qui, pris séparément, sont autonomes et complets» (Tamba, à paraître). Sa conclusion de l'article paru dans Langages est moins forte: «la spécificité du sémantique» du proverbe réside dans «la synthèse du sens littéral du probable et de son sens prescriptif lexicalisé» (Tamba 2000: 118).

14. Tamba (à paraître) a montré de façon très convaincante et stimulante que dans le cas des expressions idiomatiques (ses idiomes), il y avait analysabilité, c'est-à-dire une interprétation qui part du sens global vers les parties constituantes et non des parties au tout, comme dans la procédure compositionnelle. S’appuyant sur Nunberg, Sag et Wasow (1994), Tamba (à paraître) établit que «le sens idiomatique, bien que non compositionnel, est analysable, mais selon une procédure régressive, orientée du tout vers les éléments constitutifs dans la mesure où ce sens global se répartit plus ou moins sur les constituants locaux de l'idiome».

15. «Le sens formulaire d'un proverbe est bien un sens conventionnel, fixe et enregistré dans le lexique, tout comme le sens idiomatique» (Tamba, à paraître).

16. C'est ici qu'il faudrait placer le développement sur la structure implicative commune.

17. Voir ici les résultats obtenus par Anscombre (2000).

\section{RÉFÉRENCES}

Anscombre, Jean-Claude (1994): Proverbes et formes proverbiales: valeur évidentielle et argumentative. Langue française. 102:95-107.

Anscombre, Jean-Claude (2000): Parole proverbiale et structure métrique. Langages. 139:6-26.

Anscombre, Jean-Claude (2003): Les proverbes sont-ils des expressions figées? Cahiers de lexicologie. 82(1):159-173.

Arnaud, Pierre J. L. (1991): Réflexions sur le proverbe. Cahiers de lexicologie. 59(2):6-27. 
ARnaud, Pierre J. L. (1992): La connaissance des proverbes français par les locuteurs natifs et leur sélection didactique. Cahiers de lexicologie. 60(1):195-238.

CAREL, Marion et Schultz, Patricia (2002) : De la généricité des proverbes: une étude de L'argent ne fait pas le bonheur et Il n'y a PAS de roses sans épines. Langage et société. 102:33-70.

Conenna, Mirella (1988): Sur un lexique-grammaire comparé de proverbes. Langages. 90:99116.

Conenna, Mirella (2000): Structure syntaxique des proverbes français et italiens. Langages. 139:27-38.

Conenna, Mirella et Kleiber, Georges (2002): De la métaphore dans les proverbes. Langue française. 134:58-77.

Gouvard, Jean-Michel (1996): Les formes proverbiales. Langue française. 110:48-63.

Gross, Gaston (1996): Les expressions figées en français. Paris: Ophrys.

Honeck, Richard P. (1997): A Proverb in Mind. The Cognitive Science of Proverbial Wit and Wisdom. Mahwah: Lawrence Erlbaum Associates.

KLeIBER, Georges (1989): Sur la définition du proverbe. Recherches Germaniques. 2:233-252.

KleIBer, Georges (1990): La sémantique du prototype. Paris: PUF.

Kleiber, Georges (1994a): Nominales. Paris: Armand Colin.

Kleiber, Georges (1994b): Lexique et cognition: y a-t-il des termes de base? Rivista di Linguistica. 6(2):237-266.

Kleiber, Georges (1999a): Les proverbes; des dénominations d'un type «très très spécial». Langue française. 123:52-69.

Kleiber, Georges (1999b): Les proverbes antinomiques: une grosse pierre «logique» dans le jardin toujours «universel» des proverbes. Bulletin de la Société de Linguistique de Paris. 94(1):185-208.

Kleiber, Georges (2000a): Sur le sens des proverbes. Langages. 139:39-58.

Kleiber, Georges (2000b): Proverbes, vérité générale et dénomination. In: Martine Coene, Walter De Mulder, Patrick Dendale, et al., dir. Traiani Augusti Vestigia Pressa Sequamur. Studia Linguistica In Honorem Lilianae Tasmowski. Padova: Unipress, 705-717.

Kleiber, Georges (2000c): Proverbe: sens et dénomination. In: Gertrud Gréciano, dir. Microet macrolexèmes et leur figement discursif. Louvain: Peeters, 57-76.

Kleiber, Georges (2001): Remarques sur la dénomination. Cahiers de Praxématique. 36:21-41.

KLeiber, Georges (2002): Lexique et cognition: de la dénomination en général au proverbe en particulier. Cognitio. 11:9-37.

KLeiber, Georges (2003a): Sur la sémantique de la dénomination. Verbum. 25(1):97-106.

KLeiber, Georges (2003b): Item lexical, mots construits et polylexicalité vus sous l'angle de la dénomination. Syntaxe \& Sémantique. 5:31-46.

Kleiber, Georges (2008): Histoire de couple: proverbes et métaphores. Linguistica Investigationes. 31(2):186-199.

Kleiber, Georges (à paraître, a): Petite sémantique des proverbes. In: Actes du $25^{e}$ Congrès International de Linguistique et de Philologie Romane d'Innsbrück. (Innsbruck, 3-8 septembre 2007).

KLeIBER, Georges (à paraitre, b): Sur le chemin des proverbes: questions de classification. In: Actes du Colloque Voces y marcadores del discurso. De los connectores al argumento de autoridad (Madrid, Casa de Velazquez, 6-7 mars 2008).

Lakoff, George et Turner, Mark (1989): More than Cool Reason. A Field Guid to Poetic Metaphor. Chicago: The Chicago University Press.

MejRI, Salah (1997): Le figement lexical: descriptions linguistiques et structuration sémantique. Tunis: Publication de la Faculté des Lettres de la Manouba.

MejRI, Salah (2001): La structuration sémantique des énoncés proverbiaux. L'information grammaticale. 88:10-15.

MejRi, Salah (2006): Structure inférentielle des proverbes. In: Annelies Hacki Buhofer et Harald Burger, dir. Phraseology in Motion I. Methoden und Kritik. Proceedings zu Europhras 2004. Bâle: Hohengeheren, 175-187. 
MejRI, Salah (2009): Inférence et structuration des énoncés proverbiaux. In: Danielle LeEman, dir. Des topoï à la théorie des stéréotypes en passant par la polyphonie et l'argumentation dans la langue. Chambéry: Université de Savoie, 169-180.

Michaux, Christine (1998): Le proverbe. Vers une théorie de la parole évocative. Thèse de doctorat non publiée. Université Libre de Bruxelles.

Nunberg, Geoffrey, SAG, Ivan A. et Wasow, Thomas (1994): Idioms. Language. 70(3):491-538.

Palma, Silvia (2007): Les éléments figés de la langue. Paris: L'Harmattan.

Perrin, Laurent (2000): Remarques sur la dimension générique et sur la dimension dénominative des proverbes. Langages. 139:69-80.

Perrin, Laurent (à paraître): Idiotismes, proverbes et stéréotypes. In: Actes du Colloque Voces y marcadores del discurso. De los connectores al argumento de autoridad (Madrid, Casa de Velazquez, 6-7 mars 2008).

SCHAPIRA, Charlotte (1999): Les stéréotypes en français: proverbes et autres formules. Paris: Ophrys.

Schapira, Charlotte (2000): Proverbe, proverbialisation et déproverbialisation. Langages. 139:81-97.

SCHAPIRA, Charlotte (2002): La polyphonie dans l'énoncé gnomique: stratégies argumentatives dans la maxime. In: Marek KesıK, dir. Référence discursive dans les langues romanes et slaves. Lublin: Wydawnictwo, 233-244.

Schapira, Charlotte (2003): À la recherche du référent: la sémantique du proverbe. In: Peter Blumenthal et Jean-Emmanuel Tyvaert, dir. La cognition dans le temps. Tübingen: Niemeyer, 135-144.

TамвA, Irène (2000): Formules et dire proverbial. Langages. 139:110-118.

TAMBA, Irène (2001): Le sens métaphorique argumentatif des proverbes. Cahiers de lexicologie. 35:39-57.

TAmbA, Irène (à paraître) : Sens figé: idiomes et proverbes. In: Jean-Claude Anscombre et Salah MEJRI, dir. Le figement linguistique, la parole entravée. Paris/Genève: Honoré Champion édition.

Taylor, Archer (1931/1962): The Proverb. Pennsylvania: Hartboro.

Visetti, Yves-Marie et CADiot, Pierre (2006): Motifs et proverbes. Essai de sémantique proverbiale. Paris: PUF. 